\title{
High-throughput functional screen of mouse gastrula cDNA libraries reveals new components of endoderm and mesoderm specification
}

\author{
Eric Chiao, Jeff Leonard, Kari Dickinson, and Julie C. Baker ${ }^{1}$ \\ Department of Genetics, Stanford University Medical School, Stanford, California 94062, USA
}

\begin{abstract}
This study describes a cross-species functional screen of mouse gastrula cDNA libraries for components of endoderm and mesoderm specification. Pools of 96 cDNAs from arrayed mouse gastrula cDNA libraries were transcribed into mRNA and injected into either the presumptive mesoderm or the ectoderm of one-cell Xenopus laevis embryos. Injected embryos were examined at gastrula stage by in situ hybridization with endoderm or mesoderm markers. Using this approach, we screened over 700 pools or $\sim 60,000$ cDNAs. We identified 17 unique cDNAs that function during mesoderm and/or endoderm specification and 16 that cause general morphology changes. Identified molecules fall into eight general functional groups as follows: cell cycle components (seven), transcription factors (four), extracellular secreted molecules (seven), transmembrane receptors (one), intracellular signaling components (five), microtubule components (two), metabolism molecules (three), and unknown (four). Several of the genes we identified would not have been predicted to be involved in endoderm or mesoderm specification, highlighting the usefulness of nonbiased screening approaches. This includes Otx2, which we show is a downstream target of Xsox17 $\beta$. The speed, low cost, and high efficiency of this cross-species screen makes it an ideal method for examining cDNAs from difficult-to-obtain sources. Therefore, this approach complements the current mouse molecular genetics systems and provides a powerful means for the genome-wide examination of mammalian gene function.
\end{abstract}

[The sequence data from this study have been submitted to dbEST/GenBank under accession nos. CV736163-CV736199.]

The genomics revolution has provided biologists with the complete genome sequences of numerous organisms and a host of new technologies for the study of normal development and disease states. Tapping into genomic methodologies to understand the functional significance of genes has advanced several fields, including that of developmental biology. One window of time during mammalian development that is an ideal source for functional genomic studies is gastrulation. At this stage, which lasts only $24 \mathrm{~h}$, the embryo undergoes complex differentiation events, beginning with only a few cell types and emerging with hundreds (Hogan et al. 1986; Beddington and Robertson 1998, 1999). Simultaneously, and unique to mammals, the embryo is also undergoing massive cellular proliferation, having a cell cycle rate of $\sim 5 \mathrm{~h}$ and generating $>8000$ new cells (Snow 1977). These pivotal events make the mammalian gastrula a wealthy source to mine for key regulators of developmentally or pathologically important signaling pathways.

Due to the small size and inaccessibility of the mammalian gastrula, applying genomic approaches to elucidate gene function remains a challenge. Nevertheless, several approaches are currently being utilized to meet these challenges. The mouse is currently the most robust model for detailed molecular analysis of gene function in mammals. ENU mutagenesis, enhancer trap screens, and homologous recombination approaches have all been used to identify developmentally important genes, some as early as gastrulation (Justice et al. 1999; Anderson 2000; Mitchell et al. 2001). However, these approaches are resource intensive, time consuming, and primarily uncover loss-of-function muta-

'Corresponding author.

E-mail baker@genome.stanford.edu; fax (650) 736-1534.

Article and publication are at http://www.genome.org/cgi/doi/10.1101/ gr.2993405. tions. Important genes in functionally redundant pathways are difficult to identify, as are groups of molecules acting in concert. Recently, a large-scale in situ hybridization analysis of cDNA library clones identified potentially important developmental regulators and synexpression groups during mouse gastrulation (Sousa-Nunes et al. 2003). This approach, although clearly less resource intensive than the mutant screens, required a priori assumptions in order to increase efficiency. In any genomics approach, a reliance on such assumptions makes it unlikely that important genes with unexpected function would be found. For example, it is unlikely that a gene with an "uninteresting" expression pattern would be chosen for homologous recombination, or that a "housekeeping" gene would be selected for in situ analysis. Thus, although the approaches described above are all very effective tools for exploring gene function, they have limitations when applied to large-scale systematic genome-wide endeavors.

Signaling pathways, including those active during the formation of endoderm and mesoderm, are conserved between vertebrates, opening up the possibility of cross-species investigations of mammalian germ-layer formation (Shivdasani 2002). One very fruitful approach used to identify key vertebrate developmental regulators has been expression cloning in Xenopus (Lustig et al. 1997; Grammer et al. 2000). In this approach, mRNAs are synthesized from cDNA pools and then injected into Xenopus embryos. These embryos are then analyzed for changes caused by the protein products of the injected mRNAs. Alteration of developmental processes indicates that one or more cDNAs in the injected pool has a specified function. Pools with functional activity are subsequently split, or sib selected, until the single active clone is identified. Xenopus expression-cloning approaches have identified many key developmental molecules, including 
Smad2, Noggin, Wnt8, and Mixer, to name only a few (Smith and Harland 1991, 1992; Baker and Harland 1996; Henry and Melton 1998). The rapid macroscopic external development of Xenopus embryos coupled with the large clutch sizes makes expression cloning an ideal system for performing cost-effective high-throughput screens.

Only one study to date has used expression cloning as a high-throughput methodology (Grammer et al. 2000). That study used an arrayed neurula stage Xenopus cDNA library to screen for genes involved in apoptosis, neural development, somite formation, and blood development. By creating defined cDNA pools from their arrayed cDNA library, Grammer et. al. (2000) greatly improved the sib-selection process. Previously sib selection entailed serially diluting the cDNA pools and reinjecting the diluted pools until the single activity was found, a process that was time consuming and frequently failed. Using arrayed libraries, subpools can be created in a controlled, reproducible fashion, thereby increasing the efficiency of sib selection. Here, we adapted the high-throughput method for use in a crossspecies screen and further refined the sib-selection process so that single, active clones could be directly identified in one split. This is the first report of a high-throughput expression-cloning screen used to identify functional mammalian cDNAs, and the first such screen to target endoderm and mesoderm regulators in any species.

We have completed a high-throughput cross-species functional screen of $>60,000$ mouse gastrula cDNA clones and identified many endoderm and mesoderm specification molecules. We can categorize these molecules into eight general functional groups as follows: cell cycle components (seven), transcription factors (four), extracellular secreted molecules (seven), transmembrane receptors (one), intracellular signaling components (five), microtubule components (two), metabolism molecules (three), and unknown (four). Several of the results are unexpected and would only arise from a blind screening approach. This is apparent from our identification of two different isoforms of Fgf8, both of which induce mesoderm, and our cloning of the transcription factors Otx2 and Foxa2, both extremely wellstudied developmental molecules that have never been shown to be sufficient to induce endoderm. Here we show that Otx2 is downstream of Xsox $17 \beta$ in an endodermal specification pathway. Furthermore, this screen identified several cell cycle components that are sufficient for endodermal specification. These molecules would not have been predicted a priori to have this role, and their identification in this screen may shed light on the relationship between the rapid cellular proliferation and differentiation events occurring during mouse gastrulation. These data highlight the usefulness of nonbiased functional screening approaches for identifying new and unexpected molecules involved in the regulation of important developmental processes.

\section{Results}

To identify molecules that alter the fate of endoderm or mesoderm during mouse gastrulation, we performed a functional genomic screen using an expression-cloning approach. Two separate mouse cDNA libraries were generated, one from early stage (e6.5) and one from late stage (e7.5) gastrula (Baker et al. 1999). These cDNA libraries were arrayed on 384-well plates, and pools of 96 were generated from this resource. mRNA synthesized from these pools was injected into the presumptive mesoderm or ec- toderm of the one-cell Xenopus laevis embryo (see Fig. 1 and Methods). By overexpressing pools in the mesoderm, our goal was to find molecules that could repress or expand mesodermal or endodermal cells. By overexpressing pools in the ectoderm, our goal was to identify molecules that ectopically induce either endoderm or mesoderm. Injected embryos were allowed to develop to gastrula stage (10.5). Then, the embryos were fixed and stained by in situ hybridization for the mesodermal marker, $\mathrm{Xbra}$, or a combination of the endodermal markers, $X \operatorname{sox} 17 \beta$ and $e n$ dodermin (edd) (see Fig. 3, below). During gastrulation, the transcription factor Xbra is expressed throughout the mesoderm in a ring around the equator of the embryo (Smith et al. 1991). $X \operatorname{sox} 17 \beta$, also a transcription factor, is specifically and uniformly expressed throughout the endoderm (Hudson et al. 1997). Edd is expressed throughout the endoderm, but is expressed more strongly than $X$ sox $17 \beta$ in the dorsal bottle cells and in the rim of the blastopore (Sasai et al. 1996). We chose these markers because they are the best studied of the endoderm and mesoderm markers available and make the most reliable probes, due to their high expression within the different domains. Throughout the screen, we observed morphological phenotypes in several pools, consisting mainly of cell cycle or patterning defects. Since these effects are frequently the result of high doses of potent cell fate-inducing molecules, these pools were also examined and split.

Using this approach, we screened through 368 pools from the e6.5 library and 344 pools from the e7.5 library for a total of $\sim 60,000$ cDNAs. Eighty-two pools (11.5\%) had reproducible phenotypic effects on either endoderm and mesoderm (55) or general morphology (27). These pools were subsequently split using a gridding approach, whereby the rows and columns from the original pool are subpooled and assayed for activity. The overlap of activity reveals the single positive clone (see detailed explanation in Methods and in Fig. 2). Of the 82 positive pools, 23 single active clones were isolated by mesoderm and/or endoderm activities and 19 were isolated by general morphology changes. These clones were sequenced and their identity revealed by BLAST searches (Altschul et al. 1990). Forty-one pools either failed to split or the clone identified did not repeat the phenotypic effect reliably. Several clones were isolated more than once. A complete list of the clones identified, grouped by activity, is shown in Table 1A,B. The marker analysis of a selection of these clones is shown in Figures 3 and 4. It is interesting to point out that most of the clones were identified from the earlier staged library (e6.5). There is no indication from sequencing or clone

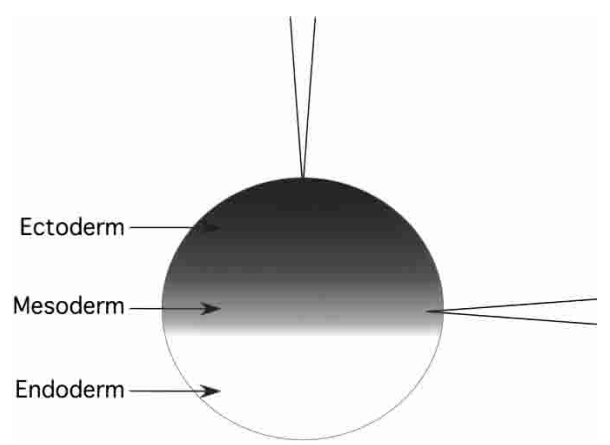

Figure 1. The Xenopus embryo is laid with a rudimentary polarity. The prospective ectoderm, (shaded) resides at the animal hemisphere, while the prospective endoderm resides at the vegetal hemisphere. The prospective mesoderm develops at the equator between these two cell types. Sites of injection are illustrated. 


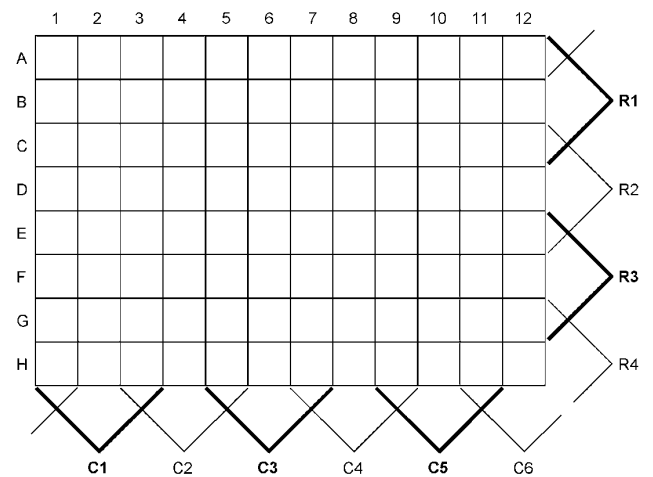

Figure 2. Positive pools were split as shown in this diagram. Ten subpools were generated of overlapping rows and columns. These subpools were injected and analyzed by in situ hybridization. Individual cDNA clones(s) within the intersection of phenotypically positive subpools were isolated and sequenced. This overlapping subpooling approach allows for the identification of positive clones in a single split.

analysis that might indicate that this is a superior library. Both appear to have similar numbers of full-length transcripts and have equivalent clone sizes.

\section{Molecules identified that induce ectopic endoderm in whole embryos}

Using this screen, we identified eight molecules that, when expressed ectopically in whole embryos, led to an increase in markers for endoderm. These include Mixl1, Hspa8, Kif22, Fzr1/Cdh1, Tcfeb, Habp2, Otx2, and Foxa2 (previously HNF3 $\beta$ ). Since this analysis consisted of a combination $X$ sox $17 \beta$ and $e d d$ probes, we characterized which of these markers the eight molecules could induce. Four molecules were identified that led to the ectopic formation of both endodermal markers-Kif22, Mixl1, Hspa8, and Fzr1/Cdh1 (Fig. 3D-O)—while four were specific for induc- tion of edd-Tcfeb, Habp2, Otx2, and Foxa2 (Fig. 3P-ZZ). None were specific for induction of $X \operatorname{sox} 17 \beta$ alone.

\section{Molecules identified that induce ectopic mesoderm in whole embryos}

We found two molecules that function as mesoderm inducers, ActrIIb, and Fgf8f. ActrIIb is a known and characterized mesoderm inducer. It is also a transmembrane receptor, which shows that the screen can recover even membrane-associated molecules (Hemmati-Brivanlou and Melton 1992; Nagaso et al. 1999). In addition, we identified another isoform of Fgf8, Fgf8a, in the gastrulation defective screen. At low concentrations, this clone can also induce mesoderm (see below and Fig. 5D).

\section{Molecules identified that disrupt mesoderm and endoderm}

We identified six molecules that disrupt mesoderm and/or endoderm without causing observable changes in cellular morphology or noticeable cellular death. The distinction between inhibition of marker expression and cellular changes is a critical one, since we wanted to be certain that inhibition was caused by a specific disruption of tissue and not a general death phenotype. Embryos injected with Rsk4, Cks2, Lefty2, cyclin F, D7Ssb2, and geminin looked phenotypically normal and healthy prior to in situ hybridization, with the exception that Lefty 2 and geminininjected embryos displayed gastrulation delays and failure of bottle cell formation. To ensure that the disruption of marker was not caused by cell death, we performed a TUNEL assay on embryos injected with several concentrations of $C k s 2$, cyclin $F$, and $D 7 S s b 2$ (data not shown). Only cyclin $F$ at concentrations $>400 \mathrm{pg}$ led to programmed cell death by gastrulation. Titration of cyclin $F$ concentrations below 400 pg inhibited Xbra in the absence of TUNEL-positive cells. These results indicate that we observe inhibition of mesoderm and endoderm in the absence of cell death. Lefty2, geminin, and D7Ssb2 inhibited both Xbra and edd expres-

Table 1A. Clones identified by endoderm or mesoderm phenotypic effect on Xenopus embryos

\begin{tabular}{|c|c|c|c|c|c|c|c|c|}
\hline \multirow[b]{2}{*}{ Gene } & \multirow[b]{2}{*}{ Group } & \multirow[b]{2}{*}{ Accession \# } & \multirow[b]{2}{*}{ MGI \# } & \multicolumn{3}{|c|}{ Molecular marker } & \multirow[b]{2}{*}{ \# cloned } & \multirow[b]{2}{*}{ Library } \\
\hline & & & & Xsox17 $\beta$ & edd & Xbra & & \\
\hline Hspa8 & un & NM_031165 & MGI:105384 & + & + & & 1 & 6.5 \\
\hline Kif22 & $\mathrm{mt}$ & NM_145588 & MGI:109233 & + & + & - & 1 & 7.5 \\
\hline Fzr1; Cdh1 & $\mathrm{cc}$ & NM_019757 & MGI:1926790 & + & + & - & 1 & 6.5 \\
\hline Mixl1 & tf & NM_013729 & MGI:1351322 & + & + & & 2 & 6.5 \\
\hline Foxa2 & tf & NM_010446 & MGI:1347476 & & + & & 1 & 7.5 \\
\hline Tcfeb & $\mathrm{tf}$ & NM_011549 & MGI:103270 & & + & - & 1 & 6.5 \\
\hline Otx2 & tf & NM_144841 & MGI:97451 & & + & - & 1 & 6.5 \\
\hline Habp2 & ex & NM_146101 & MGI:1196378 & & + & - & 1 & 7.5 \\
\hline Ccnf; cyclin F & $\mathrm{cc}$ & NM_007634 & MGI:102551 & & & - & 1 & 6.5 \\
\hline Cks2 & $\mathrm{cc}$ & NM_025415 & MGI:1913447 & & & - & 1 & 7.5 \\
\hline Rsk4 & $\mathrm{sg}$ & NM_025949 & MGI:1914321 & & & - & 1 & 6.5 \\
\hline D7Ssb2 & un & AK0002578 & MGI:1914859 & & & - & 1 & 6.5 \\
\hline Gmnn; geminin & $\mathrm{cc}$ & NM_020567 & MGI:1927344 & - & - & - & 2 & 7.5 \\
\hline Lefty2 & ex & NM_177099 & MGI:2443573 & - & - & - & 5 & $6.5(4), 7.5(1)$ \\
\hline Fgf8a & ex & NM_010205 & MGI:99604 & & & + & 1 & 6.5 \\
\hline Fgf8f & ex & ВС0 048734 & na & & & + & 1 & 6.5 \\
\hline ActRIIb & $\mathrm{tm}$ & NM_007397 & MGI:87912 & & & + & 1 & 6.5 \\
\hline
\end{tabular}

Pluses indicate ectopic expression of the listed marker in whole embryos by in situ hydridization analysis. Minuses indicate loss of expression of listed marker in whole embryos by in situ hybridization. Table includes the gene name, functional group, accession number, mouse genomic resource number (MGl; see http://www.informatics.jax.org), number of times the clone was identified and the CDNA library that produced the clone. Functional group abbreviations are as follows: (cc) cell cycle component; (tf) transcription factor; (ex) extracellular secreted molecule; (sg) intracellular signaling molecule; (tm) transmembrane protein; (mt) microtubule assembly protein; (mb) metabolic protein; (un) unknown.

\section{Genome Research} www.genome.org 
Table 1B. Clones identified in functional screen

\begin{tabular}{llllll}
\hline Gene & Group & Accession \# & MGl \# & \# cloned & Library \\
\hline Cdt1; Ris2 & cc & NM_026014 & MGl:1914427 & 2 & 6.5 \\
Cox5b & mb & NM_009942 & MGl:88475 & 1 & 6.5 \\
Pcnt2 & $\mathrm{mt}$ & NM_008787 & MGl:102722 & 1 & 6.5 \\
Apex1 & mb & NM_009687 & MGl:88042 & 1 & 7.5 \\
Rnf26 & un & NM_153762 & MGl:2388131 & 1 & 6.5 \\
Pkm2 & mb & NM_011099 & MGl:97591 & 2 & 6.5 \\
Bop1 & cc & NM_013481 & MGl:1334460 & 1 & 6.5 \\
Cdk8 & cc & NM_153599 & MGl:1196224 & 1 & 6.5 \\
2210403N09Rik & un & BC024804 & MGl:1922915 & 1 & 6.5 \\
Rhoa & sg & NM_016802 & MGl:1096342 & 1 & 6.5 \\
3 catenin & sg & NM_007614 & MGl:88276 & 1 & 6.5 \\
Wnt3a & ex & NM_009521 & MGl:98955 & 2 & 6.5 \\
Bmp2 & ex & NM_007553 & MGl:88177 & 1 & 7.5 \\
Bmp4 & ex & NM_007554 & MGl:88180 & 1 & 6.5 \\
Chn1 & sg & NM_175752 & MGl:1915674 & 1 & 7.5 \\
Racgap1 & sg & NM_012025 & MGl:1349423 & 1 & 6.5 \\
\hline
\end{tabular}

Clones identified by morphological effect on Xenopus embryos. Table includes the gene name, functional group, accession number, mouse genomic resource number (MGl; see http://www.informatics.jax.org), number of times the clone was identified, and the cDNA library that produced the clone. Functional group abbreviations are as follows: (cc) cell cycle component; (tf) transcription factor; (ex) extracellular secreted molecule; (sg) intracellular signaling molecule; (tm) transmembrane protein; (mt) microtubule assembly protein; (mb) metabolic protein; (un) unknown.

sion, while cyclin F, Cks2, and Rsk4 inhibited only Xbra (Fig. 4; see Myers et al. 2004).

\section{Molecules identified that caused morphological changes}

Cell-fate determination molecules frequently lead to morphology changes or cellular death when expressed at high levels in embryos. We identified 18 molecules that cause gastrulation defects, abnormal morphologies, or overt death (Table 1B). By diluting the concentration of these molecules, we determined which molecules led to specific cell-fate changes in germ-layer formation. Indeed, seven of these molecules, including $\beta$-catenin, RhoA, D7Ssb2, Fgf8a, Wnt3a, Bmp4, and cyclin F gave specific developmental phenotypes when diluted to a concentration that did not affect the general viability of the embryo (see Fig. 4 for D7Ssb2 and cyclin F). Interestingly, Wnt $3 a$ and Bmp4 were identified from the same pool, which led to gross embryonic death shortly after gastrulation. Splitting revealed previously described effects of both molecules; Wnt3a alone caused a duplication of embryonic axis and Bmp4 alone ventralized the embryos (Hemmati-Brivanlou and Thomsen 1995; Hatta and Takahashi 1996). $\beta$-Catenin was able to dorsalize embryos and induce a secondary axis, and RhoA inhibited the formation of optic vesicles. Both of these phenotypic effects have previously been reported (Funayama et al. 1995; Guger and Gumbiner 1995; WunnenbergStapleton et al. 1999). Fgf8a induced mesoderm at lower concentrations, while D7Ssb2 and cyclin $F$, when diluted to appropriate concentrations, inhibited the formation of mesoderm.

\section{Seven of the identified clones can directly transform naive ectoderm into endoderm and/or mesoderm}

Our screening approach used intact Xenopus embryos as a readout for molecules that could activate the expression of mesodermal or endoderm markers. Although a useful approach, it does not discriminate between molecules that can directly induce (create de novo) these tissues and molecules that will simply expand or modify the tissue already present. Therefore, we used an explant approach to test which of our identified molecules could directly induce endoderm or mesoderm from naive ectoderm (Sive et al. 2000). Mixl1, Tcfeb, Kif22, Habp2, Hspa8, Fzr1, Otx2, Foxa2, Fgf8f, and Fgf8a were all injected at different concentrations into the prospective ectoderm of the one-cell Xenopus embryo. At blastula stages (8-9), before any cell mixing, the ectoderm was excised and grown in isolation until intact siblings reached gastrula stage (10.5). The explants were harvested and analyzed with RT-PCR for marker expression. Mixl1 was capable of directly inducing both $X \operatorname{sox} 17 \beta$ and $e d d$, while Fzr1 could induce $X \operatorname{sox} 17 \beta$ (Fig. 5B). This is the first report of Fzr1, a component of the anaphase-promoting complex, being involved in endodermal specification (Visintin et al. 1997). Conversely, Mixl1 is well characterized as having this function (Lemaire et al. 1998). Tcfeb, Otx2, and Foxa2 were all able to induce edd, but not $X \operatorname{sox} 17 \beta$, consistent with the whole-embryo data (Fig. 5A,C). This indicates that they are downstream of Xsox $17 \beta$ and the farthest downstream components of the early endoderm pathway known to date. Although we consistently saw very weak $X \operatorname{sox} 17 \beta$ bands in the Kif22injected samples (data not shown), we believe that Habp2, Hspa8, and Kif22 are not able to induce endoderm from naive ectoderm. Fgf8a and Fgf8f were both potent inducers of mesoderm in naive ectoderm (Fig. 5D).

\section{Otx2 is induced by XSox17 $\beta$}

Since Otx 2 could induce $e d d$, but not $X \operatorname{sox} 17 \beta$, we wanted to determine whether it was a target of Xsox $17 \beta$. Indeed, Xenopus ectodermal explants injected with $X$ sox $17 \beta$ express Otx 2 at gastrula stages, suggesting that $O t \times 2$ is downstream of $X$ sox $17 \beta$ (Fig. $5 \mathrm{E})$.

\section{Discussion}

The clones identified in our screen can be divided into three broad categories, known regulators of endoderm or mesoderm, uncharacterized genes, and genes that have been suggested to be involved, but have not been solidly implicated. Here, we further categorize the clones we identified based upon which markers they can induce or inhibit, discuss their potential roles in either mesoderm or endoderm formation, and define where they might function during germ-layer specification.

\section{New downstream components of endoderm pathway emerge from screen}

The largest group found in this screen was composed of molecules that could induce or expand endoderm. These molecules were identified by their ability to create ectopic $X \operatorname{sox} 17 \beta$ and/or edd expression in whole gastrula. The eight clones we identified, Mixl1, Tcfeb, Kif22, Hspa8, Fzr1, Habp2, Otx2, and Foxa2, all created ectopic $e d d$ expression when injected into embryos. Only four of these, Kif22, Mixl1, Hspa8, and Fzr1 could also expand the marker $X \operatorname{sox} 17 \beta$, and some of these induced $X$ sox $17 \beta$ very weakly (see Fig. 3 ). None were capable of inducing $X$ sox $17 \beta$ without edd in the whole embryo. Since edd is downstream of $X \operatorname{sox} 17 \beta$, this result is not surprising (see Fig. 6).

Mixl1 was identified in this screen and is a well-studied member of the endodermal specification pathway (Lemaire et al. 1998; Shivdasani 2002; Stainier 2002). When overexpressed in 


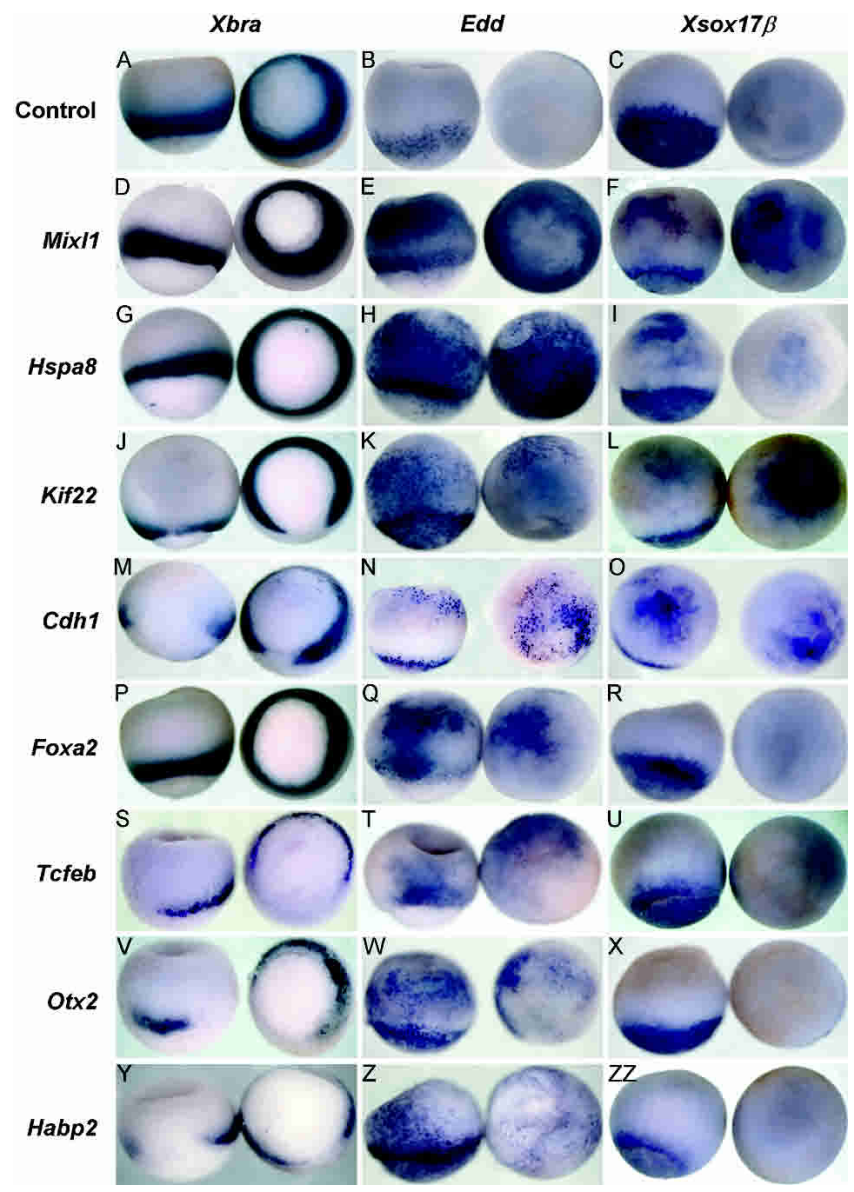

Figure 3. Positive clones that cause ectopic formation of endoderm or mesoderm in whole embryos. Gastrulating Xenopus embryos expressing positive clones were analyzed for the expression of $X b r a$ $(A, D, G, J, M, P, S, V, Y), E d d(B, E, H, K, N, Q, T, W, Z)$, or $X \operatorname{sox} 17 \beta(C, F, I, L, O$, $R, U, X, Z Z)$. Identities of clones are listed at left and include Mix/1 $(D-F)$, Hspa8 (G-l), Kif22 (J-L), Fzr1/Cdh1 (M-O), Foxa2 (P-R), Tcfeb (S-U), Otx2 $(V-X)$, and Habp2 $(Y-Z Z)$. (A-C) Uninjected controls. Concentrations of mRNAs injected into embryos are as follows: Mixl1 (D,F: $1 \mathrm{ng} ; \mathrm{E}, 333 \mathrm{pg})$, Hspa8 (G-l: $1 \mathrm{ng})$, Kif22 (J,L: 200 pg; K, 100 pg), Fzr1 (M-O: 200 pg), Foxa2 (P-R: 200 pg), Tcfeb (S: 600 pg; T,U: $1 \mathrm{ng}$ ), Otx2 (V: 500 pg; $W: 1 \mathrm{ng} ; X: 200$ pg), Habp2 (Y: 200 pg; Z: $1 \mathrm{ng} ; Z Z: 400$ pg). Embryos are oriented in Xbra column with left embryo vegetal hemisphere (endoderm) facing the bottom of the page, and right embryo vegetal hemisphere (endoderm) facing out. Embryos are oriented in both Edd and Xsox $17 \beta$ columns with left embryo vegetal hemisphere (endoderm) facing the bottom of the page and with the right embryo animal hemisphere (ectoderm) facing out.

Xenopus, it is a strong inducer of both $X \operatorname{sox} 17 \beta$ and edd. Moreover, mutations in Zebrafish, bon, a close relative, result in severe disruption of endodermal domains during early development, providing genetic evidence that the Mix family plays an important role in endoderm specification (Kikuchi et al. 2000). Therefore, the identification of Mixl1 supports the notion that we can identify important endoderm regulators using this approach.

To date, little is known about how early endoderm is specified downstream of Xsox17 $\beta$ (Shivdasani 2002; Stainier 2002; Sinner et al. 2004). The identification of molecules that can directly induce $e d d$, but not $X \operatorname{sox} 17 \beta$, is a discovery that fills a void in the downstream players of early endoderm specification (see Fig. 6). All known general endoderm specification molecules activate the expression of $X$ sox $17 \beta$. Here, we describe three mol- ecules, Tcfeb, Otx2, and Foxa2, that induce edd, but not $X \operatorname{sox} 17 \beta$, strongly suggesting that they act downstream of $X$ sox $17 \beta$. Furthermore, we show that $O t x 2$ is induced by Xsox $17 \beta$, suggesting a role directly downstream in a linear pathway. Recently, similar results were obtained for Foxa2 (Sinner et al. 2004). Although Otx2 and Foxa2 have been shown to be important for endoderm formation in the mouse, they have not been described as sufficient to induce endoderm in any vertebrate, and have not been placed in the conventional endodermal pathway (Ang et al. 1993; Dufort et al. 1998; Jin et al. 2001; Kinder et al. 2001; PereaGomez et al. 2001a,b). Tcfeb has never been described as an endoderm regulator. Together, these three molecules lay the groundwork for studying the downstream regulation of endoderm specification.

We found several molecules that have never been implicated in endoderm formation in any animal model. These include Tcfeb, Kif22, Hspa8, Frr1, and Habp2. It is yet unclear how these molecules might be involved in the pathways that specify endoderm (see Fig. 6). Tcfeb is perhaps the most "approachable" of the five, in that it is a helix-loop-helix transcription factor that has been shown to be critical for mouse embryonic survival. Homozygous mutations in mouse $T c f e b$ have defects in placental vascularization and die prior to e10.5 (Steingrimsson et al. 1998). No endodermal phenotype has been reported in these mutants. However, Tcfeb is a member of a family of closely related proteins, including Mitf, Tfe3, and Tfec, which have been shown to form heterodimers and are expressed throughout embryonic development (Steingrimsson et al. 2002). Therefore, it is plausible

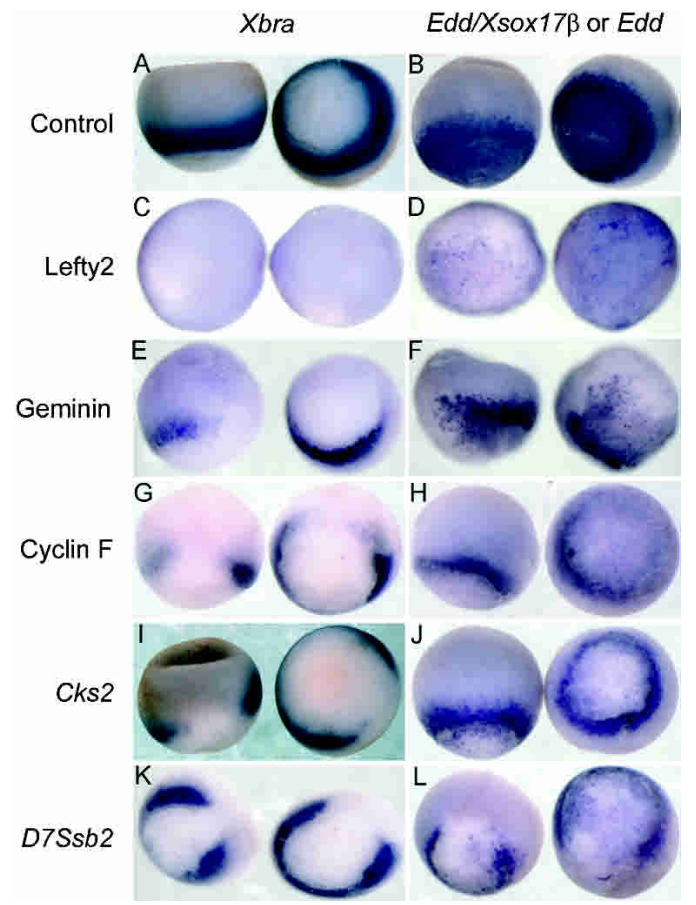

Figure 4. Positive clones that cause inhibition of mesoderm or endoderm in whole embryos. Gastrulating Xenopus embryos expressing positive clones were analyzed for the expression of $X b r a(A, C, E, G, I, K)$, a combination of edd and $X \operatorname{sox} 17 \beta(B, D, F)$ or edd alone $(H, J, L)$. Identities of clones are listed at left and include Lefty2 $(C, D)$, geminin $(E, F)$, cyclin $F$ $(G, H), C k s 2(I, J)$, and D7Ssb2 $(K, L)$. $(A, B)$ Uninjected controls. Concentrations of injected mRNAs are as follows: Lefty2 $(A, B: 1 \mathrm{ng})$, geminin ( $C, D$ : $400 \mathrm{pg})$, cyclin $F(G, H: 200 \mathrm{pg}), C k s 2(I, J: 200 \mathrm{pg})$, and D7Ssb2 (K: $10 \mathrm{ng}$; L: $500 \mathrm{pg}$ ). 
Functional screen reveals early cell-fate molecules
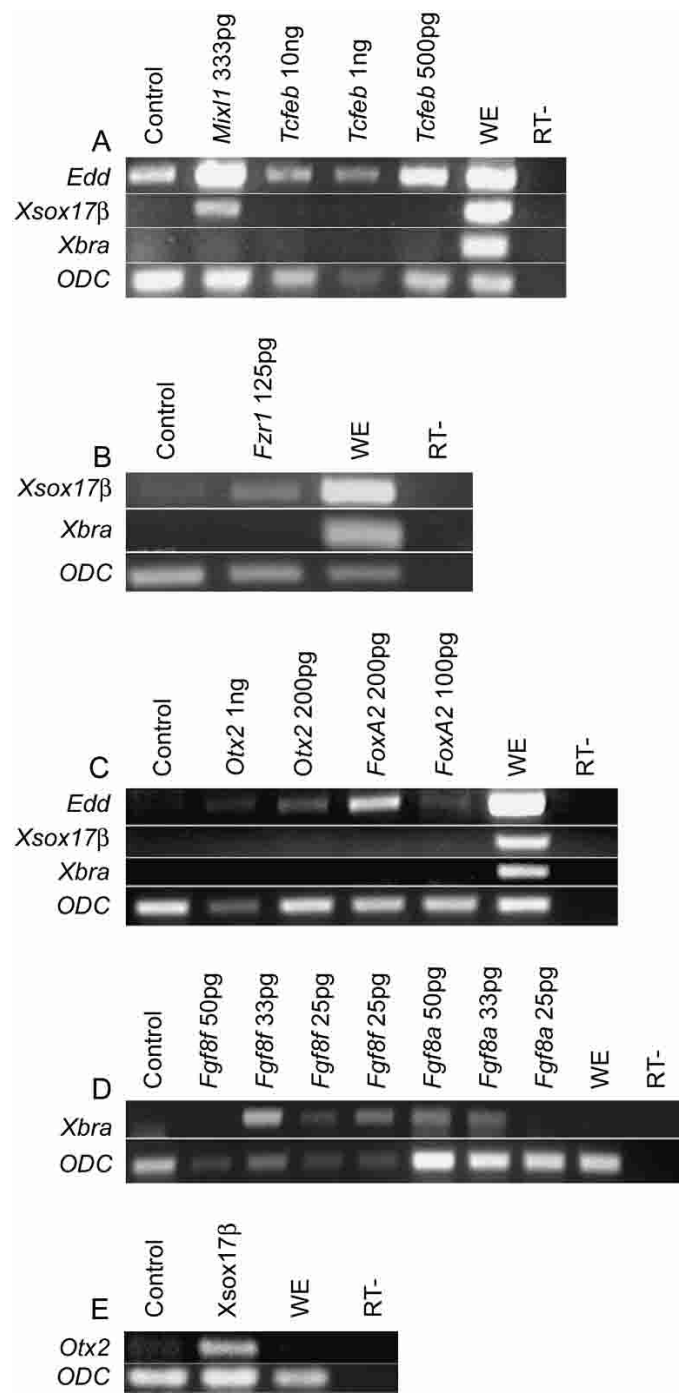

Figure 5. Identified molecules can directly induce endoderm or mesoderm from naive ectoderm. Capped synthetic mRNAs were injected at the indicated concentrations into the prospective ectoderm of the onecell Xenopus embryo. The ectoderm was explanted and cultured in isolation until stage 10.5. Analysis of mesodermal and endodermal markers was performed by RT-PCR on the expressing tissues and uninjected controls. Mix 11 was capable of directly inducing both $X \operatorname{sox} 17 \beta$ and edd, while Fzr1 could induce $X \operatorname{sox} 17 \beta$ ( $A$ and $B$ ). Tcfeb, Otx2, and Foxa2 were all able to induce edd, but not $X \operatorname{sox} 17 \beta$ ( $A$ and C). FGF8 $a$ and FGF8f both induced $X b r a$ in naive ectoderm $(D) . X$ sox $17 \beta$ induces $O t \times 2(E)$. Abbreviations are as follows: WE, whole embryo; RT-, reverse transcriptase minus. ODC is a loading control.

that an endoderm phenotype in the knock-out mouse might be masked by the redundant function of other members of this family. Double-mutant analysis is necessary to investigate this possibility. Here, we show that Tcfeb can induce naive ectoderm to form endoderm, and that its activity lies downstream of Xsox $17 \beta$. Therefore, like Foxa 2 and Otx2, Tcfeb is a new candidate for the furthest known downstream specifier of early endodermal fate.

Fzr1, in addition to its endoderm-inducing properties, generated large cells when expressed in embryos. This is consistent with its extensively characterized role as a cell cycle regulator and as an adapter protein for the anaphase-promoting complex (APC)
(Visintin et al. 1997). Fzr1/APC was shown to ubiquitinate SnoN, which is a transcriptional repressor of TGF $\beta$ downstream targets. The proteolysis of SnoN then leads to the up-regulation of TGF $\beta$ signals (Stroschein et al. 2001; Wan et al. 2001). In light of this evidence, it is not surprising that this cell cycle regulator is a direct inducer of endoderm, given that TGF $\beta$ signaling plays a critical role in endoderm formation. We show that Fzr1 induces $X$ sox $17 \beta$ in ectodermal explants, which suggests that it functions downstream of TGF $\beta$ and upstream of Xsox17 $\beta$, most likely functioning at the level of direct TGF $\beta$ target genes (Fig. 6).

Kif22, Habp2, and Hspa8 were the most intriguing molecules identified in the endodermal screen. All three can generate ectopic endoderm when overexpressed in whole embryos, but none of these can induce endoderm from naive ectoderm (with the possible exception of Kif22, which consistently generated very weak $X$ sox $17 \beta$ expression-data not shown). Kif22 is a kinesin-like protein that belongs to the very large kinesin superfamily (Miki et al. 2001). This family also includes Kif3, which has been shown to be critical for left/right symmetry in the developing vertebrate embryo (Nonaka et al. 1998). Kif22 contains an N-terminal kinesin motor and a forkhead homologyassociated domain that is thought to mediate binding to phosphoproteins. Recently, it was suggested that a kinesin motor protein plays a role in the localization of VegT and Vg1, both vegetally localized endoderm specification molecules, during oogenesis. Mislocalization of these molecules would cause the formation of ectopic endoderm, which is the effect we observe with Kif22 (Yoon and Mowry 2004). Habp2, Hyaluronanbinding-protein-2, contains a signal peptide, three EGF domains, a kringle domain, and a serine protease domain (Hashimoto et al.

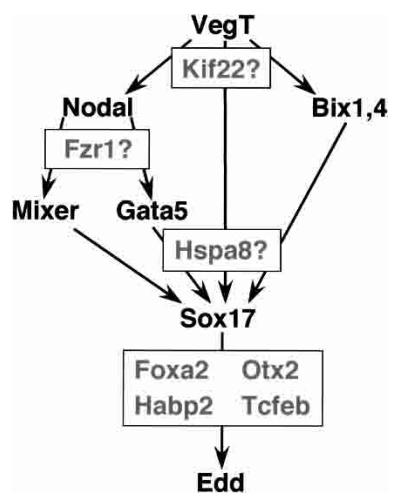

Figure 6. The endoderm specification pathway containing possible locations of identified clones. In Xenopus, the transcription factor, VegT, becomes vegetally localized during oogenesis and regulates downstream signals, including Nodal, to specify the endodermal lineage (Zhang et al. 1998; Xanthos et al. 2001). Cascades of transcription factors, forming downstream targets of Nodal, then regulate the commitment to endoderm. The Mixer-like homeodomain proteins, including Milk/Bix2, Mix.1, Bix1/Mix.4, and Bix4, and the GATA-factors, most notably Gata4 and Gata5, all activate the expression of the downstream target $X$ sox $17 \beta$ (Hudson et al. 1997; Ecochard et al. 1998; Henry and Melton 1998; Tada et al. 1998; Casey et al. 1999; Reiter et al. 1999; Weber et al. 2000; Dickmeis et al. 2001). Molecules identified in this screen have been placed in possible pathway locations. A recent report has suggested that a kinesin motor protein localizes $\mathrm{Veg} \mathrm{T}$ and $\mathrm{Vg} 1$ to the vegetal hemisphere during oogenesis; therefore, we place Kif22 near VegT (Yoon and Mowry 2004). Fzr1 acts directly downstream of Nodal (Stroschein et al. 2001; Wan et al. 2001). Foxa2, Otx2, Habp2, and Tcfeb can induce edd, but not $X \operatorname{sox} 17 \beta$, indicating that they function between these two molecules in the endoderm-specification pathway. For reviews of endoderm specification, see Stainier (2002) and Shivdasani (2002). 
1997). In the adult, Habp2 is expressed in endodermally derived organs, including the pancreas, kidney, and liver. Virtually nothing is known about the function of the vertebrate proteins, although there is a Drosophila ortholog called Masquerade (mas). Total loss of mas function is lethal, resulting in defects in the central nervous system and in muscle development (MurugasuOei et al. 1995, 1996). Hspa8 is a heat-shock protein that is highly expressed in the mouse pancreas. Little is known about this protein, other than it is not induced by heat and is thought to facilitate the correct folding of nascent polypeptides, thus acting as a chaperonin. How Kif22, Habp2, and Hspa8 can induce endoderm has yet to be determined.

While we identified Mixl1, we did not identify several of the other known endoderm specification members-including Nodal, Sox17, and Gata5. From the onset, we did not expect to find either Nodal or Sox 17, since Nodal is a rare transcript at these stages of gastrulation, and therefore not well represented in the library, and Sox17 requires large doses to induce endoderm in Xenopus. Nevertheless, it is clear that our efforts here have not yet reached screening saturation. Most of the genes we identified were found only once, and we have not yet arrayed enough independent clones from our libraries necessary for the redundancy needed to ensure full library coverage. Therefore, there remain future opportunities to use this approach to identify more novel genes involved in endoderm specification.

\section{Fgf8f and Fgf8a are mesoderm-inducing molecules}

Fibroblast growth factor (FGF) and TGF $\beta$ are the two major signaling pathways involved in the formation of mesoderm (Heasman 1997). In this screen, we have identified three molecules, Fgf8f, Fgf8a, and ActrIIb, that are components of these pathways. ActrIIb is a well-described player in mesodermal specification and patterning in mice, fish, and frogs (Hemmati-Brivanlou and Melton 1992; Nagaso et al. 1999; Song et al. 1999). Fgf8, however, is more controversial. Fgf8f and Fgf8a are members of the FGF family, which is known to be involved in mesoderm formation. However, Fgf8 has previously been reported to have distinctively different effects from the other FGFs, in that it induces neural tissue in the absence of mesoderm (Hardcastle et al. 2000; Pera et al. 2003). Therefore, we have found a different, albeit controversial, role for Fgf8 in germ-layer formation. Certainly, a role in mesoderm formation and/or maintenance is consistent with the mouse Fgf8 mutant phenotype, which has mesodermal migration defects during gastrulation (Sun et al. 1999). We have yet to determine why the $F g f f f$ and the $F g f 8$ a clones behave differently from the published reports, although multiple isoforms of Fgf8 exist and may have distinct functions.

\section{Mesoderm inhibition screen reveals new molecules}

Six molecules are described that can inhibit mesoderm in the absence of death, Rsk4, Cks2, Lefty2, geminin, D7Ssb2, and cyclin F. We were careful to distinguish during the screen between inhibition of marker expression and gross cellular abnormalities to ensure that we were looking at direct effects on the marker Xbra. Two of these molecules, Lefty 2 and Rsk4, have been characterized as inhibitors of TGF $\beta$ and FGF signaling, respectively. Lefty2 is a well-described inhibitor of TGF $\beta$ signaling and therefore inhibits the formation of endoderm and mesodermal markers (Meno et al. 1999; Cheng et al. 2000). We have recently shown that Rsk4 is a negative regulator of FGF signaling, and thus down-regulation of this pathway inhibits mesoderm locally
(Myers et al. 2004). The identification of both Lefty2 and Rsk4 provide compelling evidence that physiologically relevant molecules can be found using this approach.

D7Ssb2, cyclin F, geminin, and Cks2 were identified by their mesoderm-repressing activities. D7Ssb2 and cyclin F were originally found due to abnormal morphology changes in expressing embryos. After reducing their active concentration, we found that they could cause inhibition of mesoderm. D7Ssb2 is contained in the $75 \mathrm{Kd}$ Mesd locus in the mouse genome. This region is vital for primitive streak and mesoderm formation (Wines et al. 2001). D7Ssb2 has not been studied with respect to this effect, since it has been thought to be a pseudogene. Our sequencing results clearly show a 278 aa ORF and suggest that $D 7 S s b 2$ encodes a protein. To date, the gene residing in the Mesd locus responsible for the defect in mesoderm formation has not been identified, leaving the possibility that D7Ssb2 may contribute to this phenotypic effect.

Cyclin F, Cks2, and geminin are all regulators of the cell cycle. Very little is known about cyclin F, except that-like other cyclins-its expression is "cycled" once per cell cycle (Bai et al. 1994). Mouse mutations in cyclin $F$ have defects in placental development and in early embryonic development, particularly in the allantois, which becomes bulbous at e8.5 (Tetzlaff et al. 2004). This large allantois may be a result of an increase in posterior mesoderm, which would be consistent with our finding that cyclin $\mathrm{F}$ overexpression leads to a decrease in mesoderm. Cks2 is a Cdc Kinase subunit and is involved in the progression of cells from interphase to $\mathrm{M}$ phase during mitosis. We have yet to determine how it interferes with mesoderm formation.

Geminin is one of the few molecules known to act as a link between the control of cell proliferation and the control of differentiation. By interacting with Cdt1 and preventing its assembly onto chromatin, geminin controls cell proliferation by acting as a negative regulator of replication licensing, ensuring that DNA only replicates once (McGarry and Kirschner 1998). Geminin, however, also interacts with Hox gene products, which are essential for embryonic patterning. Recently, it has been shown that the interaction between Hox proteins and geminin can displace geminin from Cdt1, therefore promoting replication and cellular proliferation, while inhibiting gene transcription (Del Bene et al. 2004; Luo et al. 2004). These studies provide a tantalizing link between cell cycle control and differentiation. Furthermore-in support of this link-when overexpressed in Xenopus, geminin has been shown to inhibit DNA replication, as well as to act as a neurogenic molecule (Kroll et al. 1998; McGarry and Kirschner 1998; McGarry 2002). Both of these functions are distinct from the phenotypic affect we observe, which is a repression of both mesoderm and endoderm. This observation could reflect differences in activities between the frog and mouse clones, or simply be the result of concentration differences between experiments. It does not appear to be due to proliferation problems within the embryo, since the cells divide at the same rate as uninjected control embryos (data not shown). Interestingly, we also identified Cdt1 in our screen; although it does have a distinct affect on cell cycle, it does not appear to have any differentiation properties.

Although not identified by their abilities to repress mesoderm, several of the endoderm-inducing clones-including Kif22, Fzr1, Tcfeb, Otx2, and Habp2-when further analyzed, could inhibit mesoderm. Further analysis is required to analyze whether the mesoderm is transformed into endoderm or whether these are separate phenomenon.

\section{Genome Research}

www.genome.org 


\section{Conclusion}

We have completed a high-throughput cross-species functional screen of $>60,000$ mouse gastrula cDNA clones and identified endoderm and mesoderm specification molecules. The ease and low cost of collecting large numbers of gastrula stage Xenopus embryos makes this screening approach particularly useful in identifying molecules that specify early cell fates. We calculate that two people, beginning with optimized in situ probes and pools of mRNA from an arrayed library, could screen through a maximum of 60,000 cDNAs in less than 2 mo by injecting 60 pools a day. This would include a single reinjection of all pools of 96 mRNAs that showed a phenotype during the first round of injections, and takes into account the time required to split the positive pools. When optimized, this method is truly highthroughput, limited only by the availability of interesting cDNA libraries and the choice of interesting cell-fate markers. The speed, low cost, and high efficiency make this cross-species screening an ideal method for examining cDNAs from difficult to obtain sources such as the early mouse gastrula. Therefore, this approach complements the current mouse molecular genetics systems and provides a powerful means for the genome-wide examination of mammalian gene function.

\section{Methods}

\section{Construction of e6.5 and e 7.5 cDNA libraries}

E6.5 mouse embryos (291) and e7.5 (178) mouse embryos were dissected in M16 medium containing 10\% FBS. Embryonic and extraembryonic tissues were maintained in e6.5 embryos, whereas extraembryonic tissues were removed in e7.5 embryos. Embryos were homogenized using $4 \mathrm{M}$ guanadinium isothiocyanate, and total RNA isolated using acid phenol extraction (Chomczynski and Sacchi 1987). Total RNA from e6.5 (30 $\mu \mathrm{g})$ and e7.5 $(80 \mu \mathrm{g})$ embryos was used to generate cDNA using the Superscript plasmid system (Invitrogen). cDNA was cloned unidirectionally into pCS105. XL1Blue electrocompetent cells were transformed with ligated cDNAs. Each of the resulting libraries has $>500,000$ clones and an average cDNA insert size of $1.7 \mathrm{~kb}$ (Baker et al. 1999).

\section{Generation of complex mRNA pools}

The e6.5 and e7.5 cDNA libraries were arrayed on 92 and 132 384-well plates, respectively (Borchers et al. 2002). From these plates, we generated 368 pools of 96 colonies from the e6.5 library and 344 pools of 96 colonies from the e7.5 library. A 96-pin replicator was used to transfer clones from each plate onto LB plate with $100 \mu \mathrm{g} / \mathrm{mL}$ ampicillin, and plates were grown overnight at $37^{\circ} \mathrm{C}$. Colonies from each pool of 96 were scraped from the LB plate and DNA prepared with Qiagen mini-prep columns. Pooled plasmid DNA was linearized with AscI and transcribed into capped mRNA with SP6 RNA polymerase using Ambion mMessage mMachine (Ambion). The total number of individual cDNAs screened was $\sim 68,000$.

\section{Injection of Xenopus embryos with mRNA pools}

Female adult frogs were ovulated by injection of human chorionic gonadotropin (Sigma-Aldrich) and eggs were fertilized in vitro. After treatment with $2.5 \%$ cysteine $(\mathrm{pH} 8.0)$ for $5 \mathrm{~min}$ to remove jelly, embryos were placed in $1 / 3 \times$ MR (Sive et al. 2000). One-cell embryos were injected with $10 \mathrm{ng}$ of each mRNA pool into either the animal hemisphere or the marginal zone (Fig. 1). These embryos were then cultured until gastrula stages (10.25-
11) (Nieuwkoop and Faber 1994), fixed using MEMFA (0.1 M

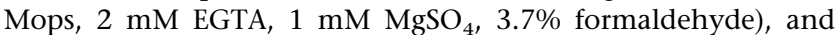
processed for in situ hybridization. Regardless of injection site, embryos were stained for both mesoderm and endodermal markers. The mesodermal marker, Xbra, was used at a concentration of $1 \mu \mathrm{g} / \mathrm{mL}$ (Smith et al. 1991). The endodermal markers, $X$ sox $17 \beta$ and $e d d$, were used as a mixture at concentrations of $1 \mu \mathrm{g} / \mathrm{mL}$ and $1.5 \mu \mathrm{g} / \mathrm{mL}$, respectively (Sasai et al. 1996; Hudson et al. 1997). In situ hybridizations were performed as previously described (Harland 1991; Sive et al. 2000). High-throughput processing was performed in 80-well Eppendorf tube racks that had been modified by replacing the bottom plastic with fine mesh and sawing off three rows, resulting in a final 65-well rack. All rinses, washes, and incubations were performed in these racks in glass Wheaton staining dishes (catalog \#900403; Wheaton). Endodermal and mesodermal marker in situ hybridization staining patterns were compared between injected and uninjected control embryos from the same clutches. Pools and identified clones were considered phenotypically positive if $>50 \%$ of the injected embryos were affected. For every phenotypically positive clone identified, in situ hybridization analysis was repeated more than three times using independent clutches of embryos.

\section{Sib selection}

Positive pools were split into 10 subpools of overlapping rows and columns, as shown in Figure 2. Subpools were injected and analyzed by in situ hybridization. If the phenotype of the original pool of 96 is due to a single cDNA within that pool, the intersection of positive subpools, in combination with the exclusion of negative subpools, defines that single cDNA. Individual cDNA clones within the intersection of phenotypically positive subpools were isolated and sequenced. The sequence was subsequently analyzed by BLAST (Altschul et al. 1990).

\section{Ectodermal explants, RT-PCR assays, and TUNEL assay}

Embryos were injected into the presumptive ectoderm at the one-cell stage, developed to stage 8-9, and transferred to $0.75 \times$ NAM solution for tissue excision (Sive et al. 2000). Explants were cultured in $0.75 \times$ NAM until stage 10.5. RNA was isolated, and reverse transcription RT-PCR was performed as previously described (Wilson and Melton 1994). Primer sequences were as follows: Xbra (F) GGATCGTTATCACCTCTG, (R) GTGTAGTCTG TAGCAGCA (Wilson and Melton 1994); ODC (F) CAGCTAGCTG TGGTGTGG (R) CAACATGGAAACTCACACC (Agius et al. 2000); Otx2 (F) CGGGATGGATTTGTTGCA, (R) TTGAACCAGACCTGG ACT; Xsox17 $\beta$ (F) AACTCCCACCAGCAGGCTACTTTG (R) TGT CAATGTCACTCTCCAGATGTCC (Myers et al. 2004); edd (F) TATTCTGACTCCTGAAGGTG (R) GAGAACTGCCCATGTGC CTC (Sasai et al. 1996).

Whole-mount TUNEL analysis of Xenopus embryos was performed as described on http://www.xenbase.org/xmmr/ Marker_pages/pcd/apoptosisTUN.htm.

\section{Acknowledgments}

We thank Kirstin Knox for thoughtful manuscript comments and help with the screen. We also thank Richard Harland for advice and support. Special thanks to Jim Smith (Xbra/pgem7), Hugh Woodland (pSPXsox17 $\beta$ ), and Eddy DeRobertis (Edd/pBS) for sharing plasmids. We acknowledge Karen Leonard for expert engineering advice on pool-splitting design and Seung Kim for advice and ideas. J.B. thanks Timothy Grammer for help in the early phases of this screen and Eric Davidson for use of Q-bot and expertise on arraying cDNA libraries. This manuscript was 
funded by the generosity of the Donald E. and Delia B. Baxter foundation, Institutional Training Grant in Genome Science (NIH HG000044), and from the National Institutes of Health (RO1 HD 41557).

\section{References}

Agius, E., Oelgeschlager, M., Wessely, O., Kemp, C., and De Robertis, E.M. 2000. Endodermal Nodal-related signals and mesoderm induction in Xenopus. Development 127: 1173-1183.

Altschul, S.F., Gish, W., Miller, W., Myers, E.W., and Lipman, D.J. 1990. Basic local alignment search tool. J. Mol. Biol. 215: 403-410.

Anderson, K.V. 2000. Finding the genes that direct mammalian development: ENU mutagenesis in the mouse. Trends Genet. 16: $99-102$.

Ang, S.L., Wierda, A., Wong, D., Stevens, K.A., Cascio, S., Rossant, J., and Zaret, K.S. 1993. The formation and maintenance of the definitive endoderm lineage in the mouse: Involvement of HNF3/forkhead proteins. Development 119: 1301-1315.

Bai, C., Richman, R., and Elledge, S.J. 1994. Human cyclin F. EMBO J. 13: 6087-6098.

Baker, J.C. and Harland, R.M. 1996. A novel mesoderm inducer, Madr2, functions in the activin signal transduction pathway. Genes \& Dev. 10: $1880-1889$.

Baker, J.C., Beddington, R.S., and Harland, R.M. 1999. Wnt signaling in Xenopus embryos inhibits bmp4 expression and activates neural development. Genes \& Dev. 13: 3149-3159.

Beddington, R.S. and Robertson, E.J. 1998. Anterior patterning in mouse. Trends Genet. 14: 277-284.

. 1999. Axis development and early asymmetry in mammals. Cell 96: 195-209.

Borchers, A.G., Hufton, A.L., Eldridge, A.G., Jackson, P.K., Harland, R.M., and Baker, J.C. 2002. The E3 ubiquitin ligase GREUL1 anteriorizes ectoderm during Xenopus development. Dev. Biol. 251: 395-408.

Casey, E.S., Tada, M., Fairclough, L., Wylie, C.C., Heasman, J., and Smith, J.C. 1999. Bix4 is activated directly by VegT and mediates endoderm formation in Xenopus development. Development 126: $4193-4200$.

Cheng, A.M., Thisse, B., Thisse, C., and Wright, C.V. 2000. The lefty-related factor Xatv acts as a feedback inhibitor of nodal signaling in mesoderm induction and L-R axis development in xenopus. Development 127: 1049-1061.

Chomczynski, P. and Sacchi, N. 1987. Single-step method of RNA isolation by acid guanidinium thiocyanate-phenol-chloroform extraction. Anal. Biochem. 162: 156-159.

Del Bene, F., Tessmar-Raible, K., and Wittbrodt, J. 2004. Direct interaction of geminin and Six3 in eye development. Nature 427: 745-749.

Dickmeis, T., Mourrain, P., Saint-Etienne, L., Fischer, N., Aanstad, P. Clark, M., Strahle, U., and Rosa, F. 2001. A crucial component of the endoderm formation pathway, CASANOVA, is encoded by a novel sox-related gene. Genes \& Dev. 15: 1487-1492.

Dufort, D., Schwartz, L., Harpal, K., and Rossant, J. 1998. The transcription factor HNF3 $\beta$ is required in visceral endoderm for normal primitive streak morphogenesis. Development 125: 3015-3025.

Ecochard, V., Cayrol, C., Rey, S., Foulquier, F., Caillol, D., Lemaire, P., and Duprat, A.M. 1998. A novel Xenopus mix-like gene milk involved in the control of the endomesodermal fates. Development 125: $2577-2585$.

Funayama, N., Fagotto, F., McCrea, P., and Gumbiner, B.M. 1995 Embryonic axis induction by the armadillo repeat domain of $\beta$-catenin: Evidence for intracellular signaling. J. Cell. Biol. 128: 959-968.

Grammer, T.C., Liu, K.J., Mariani, F.V., and Harland, R.M. 2000. Use of large-scale expression cloning screens in the Xenopus laevis tadpole to identify gene function. Dev. Biol. 228: 197-210.

Guger, K.A. and Gumbiner, B.M. 1995. $\beta$-Catenin has Wnt-like activity and mimics the Nieuwkoop signaling center in Xenopus dorsal-ventral patterning. Dev. Biol. 172: 115-125.

Hardcastle, Z., Chalmers, A.D., and Papalopulu, N. 2000. FGF-8 stimulates neuronal differentiation through FGFR-4a and interferes with mesoderm induction in Xenopus embryos. Curr. Biol. 10: $1511-1514$.

Harland, R.M. 1991. In situ hybridization: An improved whole-mount method for Xenopus embryos. Methods Cell. Biol. 36: 685-695.

Hashimoto, K., Tobe, T., Sumiya, J., Saguchi, K., Sano, Y., Nakano, Y.,
Choi-Miura, N.H., and Tomita, M. 1997. Cloning of the cDNA for a mouse homologue of human PHBP: A novel hyaluronan-binding protein. Biol. Pharm. Bull. 20: 1127-1130.

Hatta, K. and Takahashi, Y. 1996. Secondary axis induction by heterospecific organizers in zebrafish. Dev. Dyn. 205: 183-195.

Heasman, J. 1997. Patterning the Xenopus blastula. Development 124: 4179-4191.

Hemmati-Brivanlou, A. and Melton, D.A. 1992. A truncated activin receptor inhibits mesoderm induction and formation of axial structures in Xenopus embryos. Nature 359: 609-614.

Hemmati-Brivanlou, A. and Thomsen, G.H. 1995. Ventral mesodermal patterning in Xenopus embryos: Expression patterns and activities of BMP-2 and BMP-4. Dev. Genet. 17: 78-89.

Henry, G.L. and Melton, D.A. 1998. Mixer, a homeobox gene required for endoderm development. Science 281: 91-96.

Hogan, B., Costantini, F., and Lacy, E. 1986. Manipulating the mouse embryo: A laboratory manual. Cold Spring Harbor Laboratory, Cold Spring Harbor, NY.

Hudson, C., Clements, D., Friday, R.V., Stott, D., and Woodland, H.R. 1997. Xsox $17 \alpha$ and $-\beta$ mediate endoderm formation in Xenopus. Cell 91: $397-405$.

Jin, O., Harpal, K., Ang, S.L., and Rossant, J. 2001. Otx2 and HNF3 $\beta$ genetically interact in anterior patterning. Int. J. Dev. Biol 45: $357-365$.

Justice, M.J., Noveroske, J.K., Weber, J.S., Zheng, B., and Bradley, A. 1999. Mouse ENU mutagenesis. Hum. Mol. Genet. 8: 1955-1963.

Kikuchi, Y., Trinh, L.A., Reiter, J.F., Alexander, J., Yelon, D., and Stainier, D.Y. 2000. The zebrafish bonnie and clyde gene encodes a Mix family homeodomain protein that regulates the generation of endodermal precursors. Genes \& Dev. 14: 1279-1289.

Kinder, S.J., Tsang, T.E., Ang, S.L., Behringer, R.R., and Tam, P.P. 2001. Defects of the body plan of mutant embryos lacking Lim1, Otx2 or Hnf $3 \beta$ activity. Int. J. Dev. Biol. 45: 347-355.

Kroll, K.L., Salic, A.N., Evans, L.M., and Kirschner, M.W. 1998. Geminin, a neuralizing molecule that demarcates the future neural plate at the onset of gastrulation. Development 125: 3247-3258.

Lemaire, P., Darras, S., Caillol, D., and Kodjabachian, L. 1998. A role for the vegetally expressed Xenopus gene Mix.1 in endoderm formation and in the restriction of mesoderm to the marginal zone. Development 125: 2371-2380.

Luo, L., Yang, X., Takihara, Y., Knoetgen, H., and Kessel, M. 2004. The cell-cycle regulator geminin inhibits Hox function through direct and polycomb-mediated interactions. Nature 427: 749-753.

Lustig, K.D., Stukenberg, P.T., McGarry, T.J., King, R.W., Cryns, V.L., Mead, P.E., Zon, L.I., Yuan, J., and Kirschner, M.W. 1997. Small pool expression screening: Identification of genes involved in cell cycle control, apoptosis, and early development. Methods Enzymol. 283: 83-99.

McGarry, T.J. 2002. Geminin deficiency causes a Chk1-dependent G2 arrest in Xenopus. Mol. Biol. Cell 13: 3662-3671.

McGarry, T.J. and Kirschner, M.W. 1998. Geminin, an inhibitor of DNA replication, is degraded during mitosis. Cell 93: 1043-1053.

Meno, C., Gritsman, K., Ohishi, S., Ohfuji, Y., Heckscher, E., Mochida, K., Shimono, A., Kondoh, H., Talbot, W.S., Robertson, E.J., et al. 1999. Mouse Lefty2 and zebrafish antivin are feedback inhibitors of nodal signaling during vertebrate gastrulation. Mol. Cell 4: 287-298.

Miki, H., Setou, M., Kaneshiro, K., and Hirokawa, N. 2001. All kinesin superfamily protein, KIF, genes in mouse and human. Proc. Natl. Acad. Sci. 98: 7004-7011.

Mitchell, K.J., Pinson, K.I., Kelly, O.G., Brennan, J., Zupicich, J., Scherz, P., Leighton, P.A., Goodrich, L.V., Lu, X., Avery, B.J., et al. 2001. Functional analysis of secreted and transmembrane proteins critical to mouse development. Nat. Genet. 28: 241-249.

Murugasu-Oei, B., Rodrigues, V., Yang, X., and Chia, W. 1995. Masquerade: A novel secreted serine protease-like molecule is required for somatic muscle attachment in the Drosophila embryo. Genes \& Dev. 9: 139-154.

Murugasu-Oei, B., Balakrishnan, R., Yang, X., Chia, W., and Rodrigues, V. 1996. Mutations in masquerade, a novel serine-protease-like molecule, affect axonal guidance and taste behavior in Drosophila. Mech. Dev. 57: 91-101.

Myers, A.P., Corson, L.B., Rossant, J., and Baker, J.C. 2004. Characterization of mouse Rsk4 as an inhibitor of fibroblast growth factor-RAS-extracellular signal-regulated kinase signaling. Mol. Cell. Biol. 24: 4255-4266.

Nagaso, H., Suzuki, A., Tada, M., and Ueno, N. 1999. Dual specificity of activin type II receptor ActRIIb in dorso-ventral patterning during zebrafish embryogenesis. Dev. Growth Differ. 41: 119-133.

Nieuwkoop, P.D. and Faber, J. 1994. Normal table of Xenopus laevis (Daudin): A systematical and chronological survey of the development from the fertilized egg till the end of metamorphosis. Garland 
Publishing, New York.

Nonaka, S., Tanaka, Y., Okada, Y., Takeda, S., Harada, A., Kanai, Y., Kido, M., and Hirokawa, N. 1998. Randomization of left-right asymmetry due to loss of nodal cilia generating leftward flow of extraembryonic fluid in mice lacking KIF3B motor protein. Cell 95: 829-837.

Pera, E.M., Ikeda, A., Eivers, E., and De Robertis, E.M. 2003. Integration of IGF, FGF, and anti-BMP signals via Smad1 phosphorylation in neural induction. Genes \& Dev. 17: 3023-3028.

Perea-Gomez, A., Lawson, K.A., Rhinn, M., Zakin, L., Brulet, P., Mazan, S., and Ang, S.L. 2001a. Otx2 is required for visceral endoderm movement and for the restriction of posterior signals in the epiblast of the mouse embryo. Development 128: 753-765.

Perea-Gomez, A., Rhinn, M., and Ang. S.L., 2001b. Role of the anterior visceral endoderm in restricting posterior signals in the mouse embryo. Int. J. Dev. Biol. 45: 311-320.

Reiter, J.F., Alexander, J., Rodaway, A., Yelon, D., Patient, R., Holder, N., and Stainier, D.Y. 1999. Gata5 is required for the development of the heart and endoderm in zebrafish. Genes \& Dev. 13: 2983-2995.

Sasai, Y., Lu, B., Piccolo, S., and De Robertis, E.M. 1996. Endoderm induction by the organizer-secreted factors chordin and noggin in Xenopus animal caps. EMBO J. 15: 4547-4555.

Shivdasani, R.A. 2002. Molecular regulation of vertebrate early endoderm development. Dev. Biol. 249: 191-203.

Sinner, D., Rankin, S., Lee, M., and Zorn, A.M. 2004. Sox17 and $\beta$-catenin cooperate to regulate the transcription of endodermal genes. Development 131: 3069-3080.

Sive, H.L., Grainger, R.M., and Harland, R.M. 2000. Early development of Xenopus laevis: A laboratory manual. Cold Spring Harbor Laboratory Press, Cold Spring Harbor, NY.

Smith, W.C. and Harland, R.M. 1991. Injected Xwnt-8 RNA acts early in Xenopus embryos to promote formation of a vegetal dorsalizing center. Cell 67: 753-765.

. 1992. Expression cloning of noggin, a new dorsalizing factor localized to the Spemann organizer in Xenopus embryos. Cell 70: $829-840$

Smith, J.C., Price, B.M., Green, J.B., Weigel, D., and Herrmann, B.G. 1991. Expression of a Xenopus homolog of Brachyury (T) is an immediate-early response to mesoderm induction. Cell 67: 79-87.

Snow, M.H.L. 1977. Gastrulation in the mouse: Growth and regionalization of the epiblast. J. Embryol. Exp. Morphol. 42: 293303.

Song, J., Oh, S.P., Schrewe, H., Nomura, M., Lei, H., Okano, M., Gridley, T., and Li, E. 1999. The type II activin receptors are essential for egg cylinder growth, gastrulation, and rostral head development in mice. Dev. Biol. 213: 157-169.

Sousa-Nunes, R., Rana, A.A., Kettleborough, R., Brickman, J.M., Clements, M., Forrest, A., Grimmond, S., Avner, P., Smith, J.C., Dunwoodie, S.L., et al. 2003. Characterizing embryonic gene expression patterns in the mouse using nonredundant sequence-based selection. Genome Res. 13: 2609-2620.

Stainier, D.Y. 2002. A glimpse into the molecular entrails of endoderm formation. Genes \& Dev. 16: 893-907.

Steingrimsson, E., Tessarollo, L., Reid, S.W., Jenkins, N.A., and Copeland, N.G. 1998. The bHLH-Zip transcription factor Tfeb is essential for placental vascularization. Development 125: 4607-4616.

Steingrimsson, E., Tessarollo, L., Pathak, B., Hou, L., Arnheiter, H., Copeland, N.G., and Jenkins, N.A. 2002. Mitf and Tfe3, two members of the Mitf-Tfe family of bHLH-Zip transcription factors, have important but functionally redundant roles in osteoclast development. Proc. Natl. Acad. Sci. 99: 4477-4482.

Stroschein, S.L., Bonni, S., Wrana, J.L., and Luo, K. 2001. Smad3 recruits the anaphase-promoting complex for ubiquitination and degradation of SnoN. Genes \& Dev. 15: 2822-2836.

Sun, X., Meyers, E.N., Lewandoski, M., and Martin, G.R. 1999. Targeted disruption of Fgf8 causes failure of cell migration in the gastrulating mouse embryo. Genes \& Dev. 13: 1834-1846.

Tada, M., Casey, E.S., Fairclough, L., and Smith, J.C. 1998. Bix1, a direct target of Xenopus T-box genes, causes formation of ventral mesoderm and endoderm. Development 125: 3997-4006.

Tetzlaff, M.T., Bai, C., Finegold, M., Wilson, J., Harper, J.W., Mahon, K.A., and Elledge, S.J. 2004. Cyclin F disruption compromises placental development and affects normal cell cycle execution. Mol. Cell. Biol. 24: 2487-2498.

Visintin, R., Prinz, S., and Amon, A. 1997. CDC20 and CDH1: A family of substrate-specific activators of APC-dependent proteolysis. Science 278: $460-463$

Wan, Y., Liu, X., and Kirschner, M.W. 2001. The anaphase-promoting complex mediates TGF- $\beta$ signaling by targeting SnoN for destruction. Mol. Cell 8: 1027-1039.

Weber, H., Symes, C.E., Walmsley, M.E., Rodaway, A.R., and Patient, R.K. 2000. A role for GATA5 in Xenopus endoderm specification. Development 127: 4345-4360.

Wilson, P.A. and Melton, D.A. 1994. Mesodermal patterning by an inducer gradient depends on secondary cell-cell communication. Curr. Biol. 4: 676-686.

Wines, M.E., Lee, L., Katari, M.S., Zhang, L., DeRossi, C., Shi, Y., Perkins, S., Feldman, M., McCombie, W.R., and Holdener, B.C. 2001. Identification of mesoderm development (mesd) candidate genes by comparative mapping and genome sequence analysis. Genomics 72: 88-98.

Wunnenberg-Stapleton, K., Blitz, I.L., Hashimoto, C., and Cho, K.W. 1999. Involvement of the small GTPases XRhoA and XRnd1 in cell adhesion and head formation in early Xenopus development. Development 126: 5339-5351.

Xanthos, J.B., Kofron, M., Wylie, C., and Heasman, J. 2001. Maternal VegT is the initiator of a molecular network specifying endoderm in Xenopus laevis. Development 128: 167-180.

Yoon, Y.J. and Mowry, K.L. 2004. Xenopus Staufen is a component of a ribonucleoprotein complex containing Vg1 RNA and kinesin. Development 131: 3035-3045.

Zhang, J., Houston, D.W., King, M.L., Payne, C., Wylie, C., and Heasman, J. 1998. The role of maternal VegT in establishing the primary germ layers in Xenopus embryos. Cell 94: 515-524.

\section{Web site references}

http://www.xenbase.org/xmmr/Marker_pages/pcd/apoptosisTUN.htm; A database pertaining to the cell and developmental biology of the frog, Xenopus.

http://www.informatics.jax.org; Provides integrated access to data on the genetics, genomics, and biology of the laboratory mouse.

Received July 13, 2004; accepted in revised form October 13, 2004. 


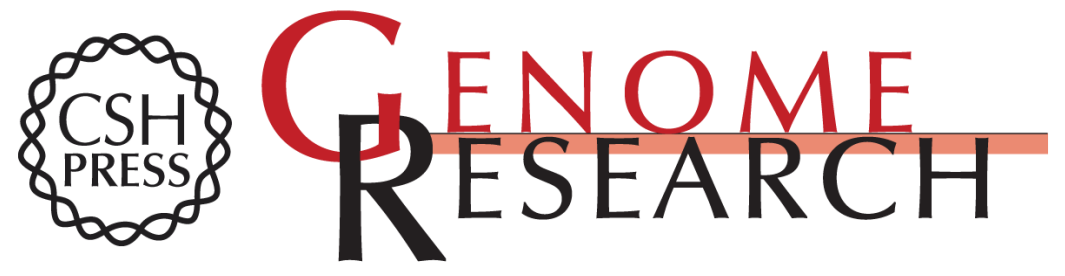

\section{High-throughput functional screen of mouse gastrula cDNA libraries reveals new components of endoderm and mesoderm specification}

Eric Chiao, Jeff Leonard, Kari Dickinson, et al.

Genome Res. 2005 15: 44-53

Access the most recent version at doi:10.1101/gr.2993405

References This article cites 76 articles, 36 of which can be accessed free at:

http://genome.cshlp.org/content/15/1/44.full.html\#ref-list-1

\section{License}

Email Alerting

Receive free email alerts when new articles cite this article - sign up in the box at the Service top right corner of the article or click here.

\section{Affordable, Accurate Sequencing.}

To subscribe to Genome Research go to: https://genome.cshlp.org/subscriptions 